\title{
Inhibitory effects of proton beam irradiation on integrin expression and signaling pathway in human colon carcinoma HT29 cells
}

\author{
BYUNG GEUN HA ${ }^{1}$, JUNG-EUN PARK ${ }^{1}$, HYUN-JUNG CHO ${ }^{1}$, YOUNG-BIN LIM ${ }^{2}$ and YUN HEE SHON ${ }^{1}$ \\ ${ }^{1}$ Bio-Medical Research Institute, Kyungpook National University Hospital, Daegu; ${ }^{2}$ Division of Radiation Effects, \\ Korea Institute of Radiological and Medical Sciences, Seoul, Republic of Korea
}

Received January 30, 2015; Accepted March 12, 2015

DOI: $10.3892 /$ ijo.2015.2942

\begin{abstract}
Proton radiotherapy has been established as a highly effective modality used in the local control of tumor growth. Although proton radiotherapy is used worldwide to treat several types of cancer clinically with great success due to superior targeting and energy deposition, the detailed regulatory mechanisms underlying the functions of proton radiation are not yet well understood. Accordingly, in the present study, to assess the effects of proton beam on integrinmediated signaling pathways, we investigated the expression of integrins related to tumor progression and integrin trafficking, and key molecules related to cell adhesion, as well as examining phosphorylation of signaling molecules involved in integrin-mediated signaling pathways. Proton beam irradiation inhibited the increase in 12- $O$-tetradecanoylphorbol-13-acetate (TPA)-induced integrin $\beta 1$ protein expression and the gene expression of members of the integrin family, such as $\alpha 5 \beta 1$, $\alpha 6 \beta 4, \alpha v \beta 3$, and $\alpha v \beta 6$ in human colorectal adenocarcinoma HT-29 cells. Simultaneously, the gene expression of cell adhesion molecules, such as FAK and CDH1, and integrin trafficking regulators, such as RAB4, RAB11, and HAX1, was decreased by proton beam irradiation. Moreover, proton beam irradiation decreased the phosphorylation of key molecules involved in integrin signaling, such as FAK, Src, and p130Cas, as well as PKC and MAPK, which are known as promoters
\end{abstract}

Correspondence to: Professor Yun Hee Shon, Bio-Medical Research Institute, Kyungpook National University Hospital, 44-2 Samduk 2ga, Jung-gu, Daegu 700-721, Republic of Korea

E-mail: yhshon@hmail.knu.ac.kr

Abbreviations: TPA, 12-O-tetradecanoylphorbol-13-acetate; FAK, focal adhesion kinase; AMPK, AMP-activated protein kinase; PKC, protein kinase C; MAPK, mitogen-activated protein kinases; ECM, extracellular matrix; Rab GTPase, Ras-associated binding small GTPase; Rab IP4, Rab4 effector protein; HAX1, HS1-associated protein $\mathrm{X} 1$; PBT, proton beam therapy

Key words: proton radiotherapy, integrin $\beta 1$, integrin trafficking, colorectal adenocarcinoma, focal adhesion kinase, AMP-activated protein kinase of cell migration, while increased the phosphorylation of AMPK and the gene expression of Rab IP4 involved in the inhibition of cell adhesion and cell spreading. Taken together, our findings suggest that proton beam irradiation can inhibit metastatic potential, including cell adhesion and migration, by modulating the gene expression of molecules involved in integrin trafficking and integrin-mediated signaling, which are necessary for tumor progression.

\section{Introduction}

Tumor invasion and metastasis are the main biological characteristics of malignant cancers. Mortality in cancer patients principally results from the metastatic spread of cancer cells to distant organs. Tumor metastasis is a highly complex and multistep process, which includes changes in cell-cell adhesion properties. A number of molecules, including matrix metalloproteinases (MMPs) (1), integrins (2), and Rac GTPases (3), have been found to be responsible for cancer cell motility.

Alterations in integrin-mediated signaling pathways and integrin trafficking are involved in nearly all steps of carcinogenesis including adhesion and migration, which include changes in the utilization of $\alpha \beta$ heterodimers, aberrant expression of integrins, and constitutive activation of downstream molecules of integrin signaling pathways (4). Integrins play important roles in pathological angiogenesis and tumor metastasis, making them attractive targets for cancer therapy strategies (5). Integrins $\alpha 5 \beta 1, \alpha 6 \beta 4, \alpha v \beta 3$, and $\alpha v \beta 6$ have been extensively studied in cancer and their expressions are closely associated with cancer progression in various tumor types (6). Upregulation of these integrins renders cancer cells more motile, invasive, and resistant to anticancer drugs (7). Integrins transmit signals across the plasma membrane via the tyrosine kinases Src and focal adhesion kinase (FAK) and the CRK-associated tyrosine kinase substrate p130Cas, and thereby regulate cell adhesion, migration, invasion, proliferation, and differentiation (8). In addition, numerous studies have indicated that many signaling molecules, including AMP-activated protein kinase (AMPK) $(9,10)$, protein kinase $\mathrm{C}$ (PKC) (11), and mitogen-activated protein kinases (MAPK) (12), are associated with integrin-mediated regulation of metastasis in cancer cells. 
Integrin trafficking regulates cell adhesion to extracellular matrix (ECM), establishes and maintains cell polarity, redefines signaling pathways, and controls migration (13). It is regulated by members of the Ras-associated binding (Rab) family of small GTPases, which function as molecular switches regulating vesicular transport in eukaryotic cells. Rab11 mediates slow integrin recycling through recycling endosomes, whereas Rab4 mediates fast integrin recycling directly from early endosomes (14). The deregulation of Rab GTPases is closely related to cancer development and progression (15). Because of the involvement of Rab4 in the recycling of $\alpha v \beta 3$ integrin, inhibition of Rab4 effector protein (Rab IP4) blocks integrin recycling, leading to inhibition of cell adhesion and cell spreading (16). Integrin $\alpha v \beta 6$ is internalized by a clathrin-dependent mechanism by interaction with HS1-associated protein X1 (HAX1). HAX1 is found in clathrin-coated vesicles. When the cytodomain of $\beta 6$ integrin interacts with HAX1 and is endocytosed, carcinoma migration and invasion is increased (17).

Heavy-particle radiotherapy, including the use of protons and carbon ions, has been producing noteworthy clinical results worldwide (18). However, the detailed regulatory mechanisms underlying their functions are not yet well understood. In our previous studies $(19,20)$, we demonstrated that proton beam irradiation suppresses metastatic capabilities such as migration, invasion, and MMP-2 and -9 expression, as well as increasing chemopreventive enzymes such as quinone reductase (QR) and glutathione S-transferase (GST) in human colorectal adenocarcinoma HT-29 cells. In the present study, to elucidate the regulatory mechanisms underlying the inhibitory effect of proton beam irradiation on metastatic potential, we investigated the effects of proton beam on the expression of members of the integrin family and trafficking regulators, and integrin signaling pathways related to tumor progression.

\section{Materials and methods}

Materials. The following items were purchased from the stated commercial sources: $12-O$-tetradecanoyl phorbol13-acetate (TPA) from Sigma-Aldrich Co. (St. Louis, MO, USA); mouse anti-human FAK (pY397), rabbit anti-phospho Src (Tyr416), rabbit anti-phospho p130Cas (Tyr410), rabbit anti-phospho AMPKa (Thr172), and rabbit anti-phospho PKC (pan) ( $\zeta$ Thr410) from Cell Signaling Technology (Danvers, MA, USA); mouse anti-human phospho MAPK (Tyr204), mouse anti- $\beta$-actin, horseradish peroxidase (HRP)-conjugated anti-mouse IgG, and anti-rabbit IgG-HRP antibodies from Santa Cruz Biotechnology Inc. (Santa Cruz, CA, USA); ECL Plus Western Blotting Substrate from Pierce Biotechnology (Rockford, IL, USA); TRIzol from Invitrogen Life Technologies (Carlsbad, CA, USA); PrimeScript ${ }^{\mathrm{TM}}$ 1st strand cDNA Synthesis kit from Takara Bio Inc. (Shiga, Japan); FastStart Universal SYBR Green Master from Roche Applied Science (Mannheim, Germany); phosphatase inhibitor cocktail and protease inhibitor cocktail solutions from GenDEPOT (Barker, TX, USA).

Cell culture. The human colon adenocarcinoma cell line, HT-29, was obtained from the Korean Cell Line Bank (KCLB no. 30038 , Seoul, Korea). Cells were grown in $5 \% \mathrm{CO}_{2}$ at $37^{\circ} \mathrm{C}$ in RPMI-1640 medium supplemented with $10 \%$ fetal bovine serum, penicillin, and streptomycin. To induce metastatic potential, cells were incubated with $150 \mathrm{nM}$ TPA for $1 \mathrm{~h}$ before proton beam irradiation.

Proton beam irradiation. Cell irradiation with a $35-\mathrm{MeV}$ proton beam using the MC-50 cyclotron (Scanditronix, Uppsala, Sweden) was carried out at the Korean Institute of Radiological and Medical Sciences (Seoul, Korea) according to a previous study (21). Cells anchored in a $12.5-\mathrm{cm}^{2}$ flask filled with medium were placed on a beam stage and then irradiated. Cells were irradiated (0.5, 2, 8 and 16 Gy) at the center of Bragg peaks modulated to 6-cm width. Flasks were oriented such that the growth surface was orthogonal to the horizontal beam entering from the top of the flask. A mono-energetic proton beam cannot be applied for cancer cells because the Bragg peak is too narrow to give a uniform dose to a tumor of any significant depth. Thus, a region of high dose uniformity in the percent depth-dose, known as spread-out Bragg-peak (SOBP) dose distribution was created by traversing a rotating range modulator designed to obtain a uniform dose distribution to an indicated depth in cells plated and the media. It was assumed that the thickness of the cell monolayer was between 3-6 $\mu \mathrm{m}$ and that of media was $1 \mathrm{~cm}$. Thus dose distribution by SOBP was enough to target live cells. The average dose rate was $2.31 \mathrm{~Gy} / \mathrm{sec}$. Radiochromic film (GAF-MD55) was used as an in situ measuring tool of the dose at each beam irradiation.

Western blot analysis. After irradiation, cells were incubated for 1 and 3 days, washed with ice-cold PBS, and lysed in RIPA buffer $(50 \mathrm{mM} \mathrm{NaCl}, 10 \mathrm{mM}$ Tris, $0.1 \%$ SDS, $1 \%$ Triton X-100, 0.1\% sodium deoxycholate, $5 \mathrm{mM}$ EDTA, and $1 \mathrm{mM} \mathrm{Na}_{3} \mathrm{VO}_{4}, \mathrm{pH}$ 7.4). Proteins $(40 \mu \mathrm{g})$ were separated by SDS-polyacrylamide gel electrophoresis and transferred to nitrocellulose membranes (Whatman, Dassel, Germany). The membranes were blocked with 5\% skimmed milk for $1 \mathrm{~h}$ and incubated with primary antibody (diluted 1:1,000) overnight at $4^{\circ} \mathrm{C}$. After washing with Tris-buffered saline containing $0.1 \%$ Tween-20, the membranes were incubated with HRP-conjugated secondary antibodies (diluted 1:3,000) for $1 \mathrm{~h}$ at room temperature. Antibodies binding on the nitrocellulose membranes were detected with an enhanced chemiluminescence solution (Amersham Bioscience, Buckinghamshire, UK) and radiography. The images were obtained with a Lumino image analyzer (LAS-4000 Mini, Fujifilm, Tokyo, Japan) and analyzed with image analysis software (Multi Gauge ver. 3.0, Fujifilm).

Quantitative RT-PCR ( $q R T-P C R)$ analysis. Total RNA was isolated from HT-29 using TRIzol (Invitrogen Life Technologies), and cDNA was synthesized using PrimeScript ${ }^{\mathrm{TM}}$ 1st strand cDNA synthesis kit (Takara Bio Inc.), according to the instructions of the manufacturer. qRT-PCR was performed in triplicate using a FastStart SYBR Green Master Mix (Roche Diagnostics, Mannheim, Germany) in ABI PRISM 7300 Sequence Detection System (Applied Biosystems, Foster City, CA, USA). The expression levels of target genes relative to that of the endogenous reference gene, actin, were calculated using the delta cycle threshold method (22) (Table I). 
Table I. Primers for quantitative RT-PCR analysis.

Forward

Reverse

\begin{tabular}{lll}
\hline ITGA5 & GGCAGCTATGGCGTCCCACTGTGG & GGCATCAGAGGTGGCTGGAGGCTT \\
ITGA6 & GGAGCCCCACAGTATTTGA & TTCCATTTGCAGATCCATGA \\
ITGAV & ACTCAAGCAAAAGGGAGCAA & TGCAAGCCTGTTGTATCAGC \\
ITGB1 & AATGAAGGGCGTGTTGGT & CTGCCAGTGTAGTTGGGGTT \\
ITGB3 & CGTCCAGGTCACCTTTGATT & GTGGCAGACACATTGACCAC \\
ITGB4 & ATGAGGCCTGAGAAGCTGAA & GCTGACTCGGTGGAGAAGAC \\
ITGB6 & TGCGACCATCAGTGAAGAAG & GTAGGACAACCCCGATGAGA \\
FAK & TGGTGAAAGCTGTCATCGAG & CTGGGCCAGTTTCATCTTGT \\
CDH1 & TGCCCAGAAAATGAAAAAGG & GGATGACACAGCGTGAGAGA \\
RAB 4 & CACTCGAGCAATGTCCGAAACCTACG & GTGAATTCCTAACAACCACACTCCTGAGC \\
RAB 11 & CACTCGAGCAATGGGCACCCGCGACGAC & GTGAATTCCTTAGATGTTCTGACAGCAC \\
HAX1 & ATGGACCCCCATCCTAGAAC & CTGCTATCTGCTTCGTGTCG \\
Rab IP4 & CCTTTGGAACTGGTGGAGAA & ACCAGCAGCCCAACAATTAC
\end{tabular}

ITGA5, integrin $\alpha 5$; ITGA6, integrin $\alpha 6$; ITGAV, integrin $\alpha$; ITGB 1 , integrin $\beta 1$; ITGB3, integrin $\beta 3$; ITGB4, integrin $\beta 4$; ITGB6, integrin $\beta 6$; FAK, focal adhesion kinase; CDH1, E-cadherin; RAB 4, RAS-related GTP-binding protein 4; RAB 11, RAS-related GTP-binding protein 11; HAX1, HS1-associated protein X1; Rab IP4, Rab4 effector protein.

Statistical analysis. All data are presented as the mean \pm SEM. The data were evaluated by one-way analysis of variance (ANOVA). Differences between the mean values were assessed using Dunnett's multiple comparisons test. Statistical significance was defined as $\mathrm{P}<0.05$.

\section{Results}

The inhibitory effect of proton beam irradiation on the TPA-induced integrin $\beta 1$ expression. To determine whether proton beam irradiation regulates the expression of integrin $\beta 1$, we investigated the expression of integrin $\beta 1$ in TPA-induced aggressive HT-29 human colorectal adenocarcinoma cells after the cells were irradiated by proton beam at 0.5, 2, 8 and $16 \mathrm{~Gy}$. The treatment of TPA for $1 \mathrm{~h}$ resulted in higher expression of integrin $\beta 1$ than that of non-treated control, while proton beam irradiation severely inhibited TPA-induced integrin $\beta 1$ expression in a dose-dependent manner $12 \mathrm{~h}$ (Fig. 1A) and $24 \mathrm{~h}$ (Fig. 1B) after irradiation. Twelve hours after proton beam irradiation, the dose of $16 \mathrm{~Gy}$ showed the strongest inhibitory effect on the expression of integrin $\beta 1$. Therefore, these findings suggest that proton beam can inhibit metastatic potential, including migration and invasion, in TPA-induced HT-29 human colorectal adenocarcinoma cells.

Proton beam irradiation inhibits TPA-induced gene expressions of integrins involved in migration and invasion. To further explore the effect of proton beam irradiation on integrin expression, we next investigated the gene expressions of integrin subunits, such as ITGA5 and ITGB1 $(\alpha 5 \beta 1)$ that form a fibronectin receptor, ITGA6 and ITGB4 $(\alpha 6 \beta 4)$ that form a laminin receptor, ITGAV and ITGB3 ( $\alpha$ v $\beta 3)$ that form fibronectin and vitronectin receptors, and ITGAV and ITGB3 $(\alpha v \beta 6)$. The expressions of these subunits have been extensively shown to be correlated with cancer progression in
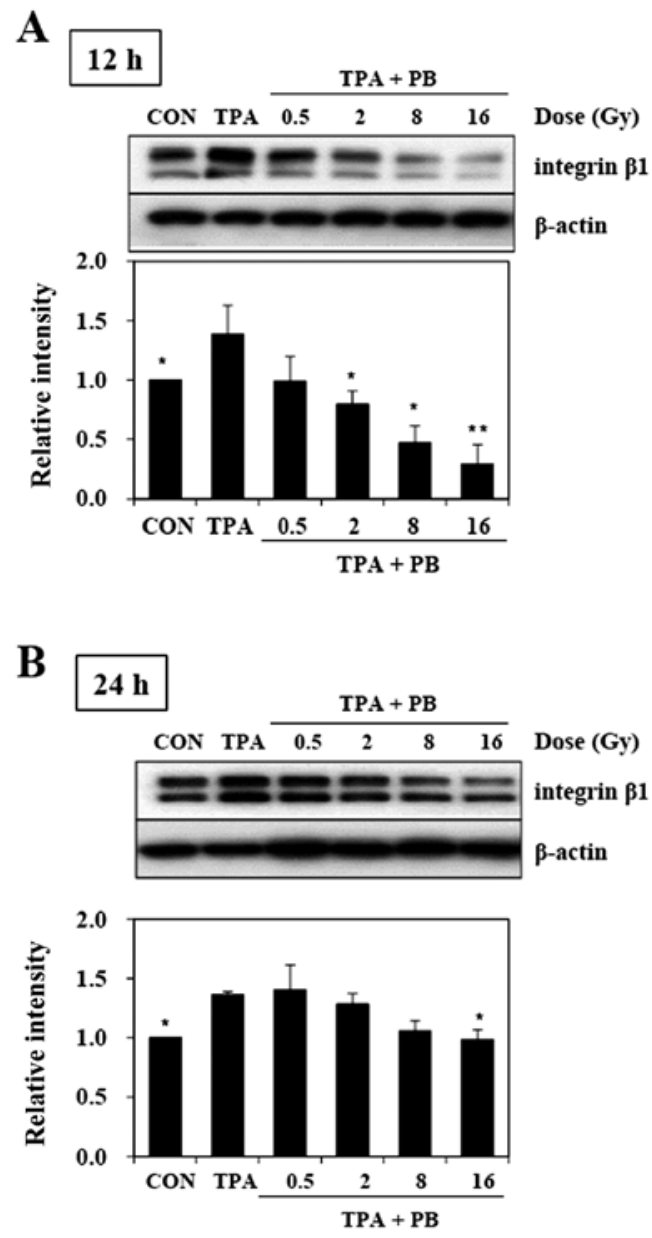

Figure 1. Effects of proton beam irradiation on the expression of integrin $\beta 1$ in TPA-induced HT-29 human colorectal adenocarcinoma cells. Before irradiation, cells were treated with $150 \mathrm{nM}$ TPA for $1 \mathrm{~h}$. Expression of integrin $\beta 1$ was determined by western blot analysis at $12 \mathrm{~h}$ (A) and $24 \mathrm{~h} \mathrm{(B)}$ after irradiation with indicated doses. $\beta$-actin served as the loading control Each value represents the mean \pm SEM of three independent experiments ${ }^{*} \mathrm{P}<0.05,{ }^{* *} \mathrm{P}<0.01$ vs. the TPA group. 

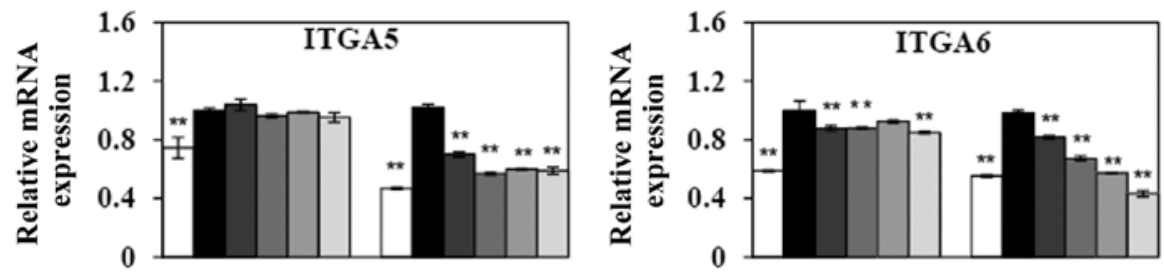

$12 \mathrm{~h} \quad 24 \mathrm{~h}$
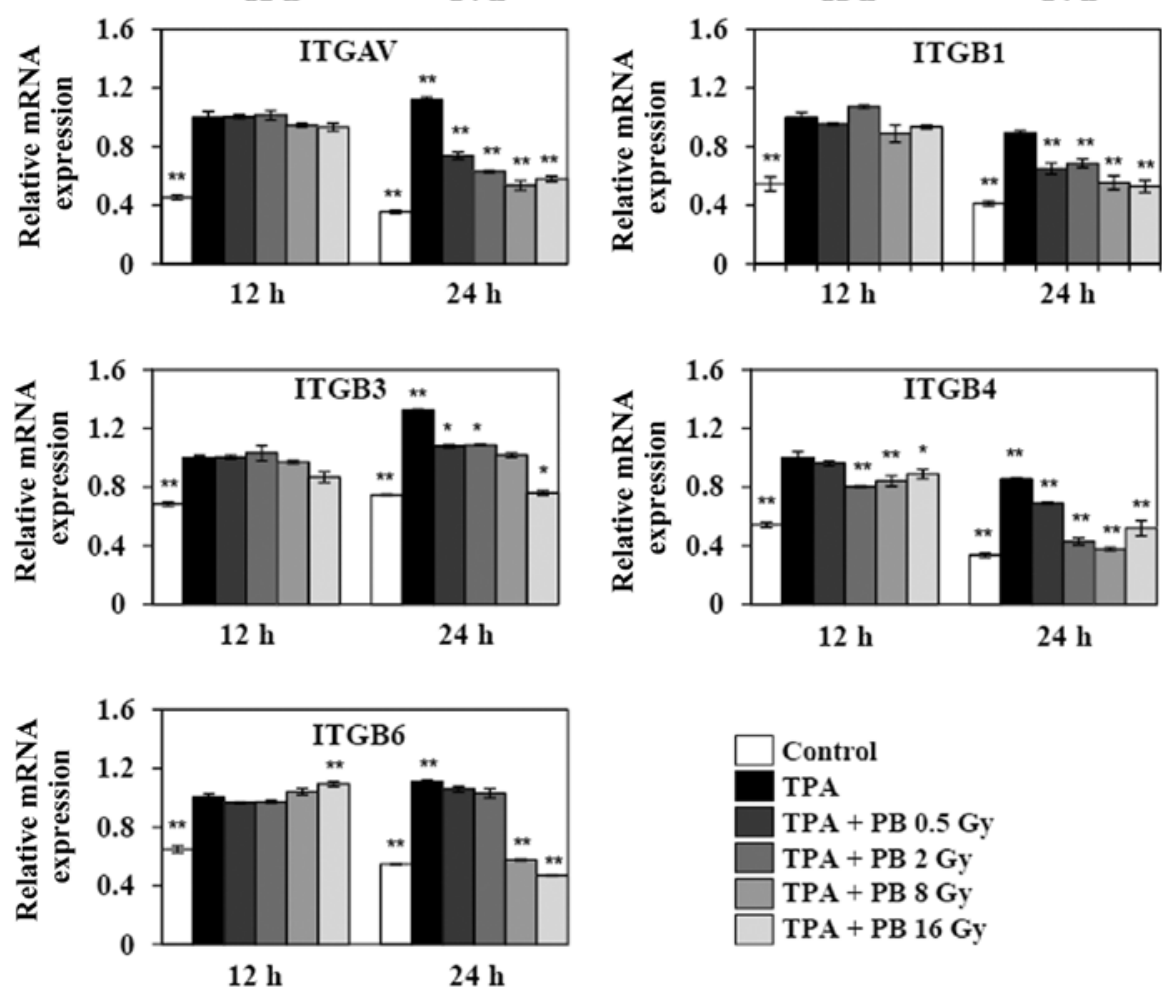

Figure 2. Effects of proton beam irradiation on the gene expression of members of the integrin family involved in tumor progression in TPA-induced HT-29 human colorectal adenocarcinoma cells. Before irradiation, cells were treated with $150 \mathrm{nM}$ TPA for $1 \mathrm{~h}$. After cells were irradiated with proton beam at different doses, expression of $\alpha 5 \beta 1$ (ITGA5 and ITGB1) for fibronectin receptor, $\alpha 6 \beta 4$ (ITGA6 and ITGB4) for laminin receptor, $\alpha v \beta 3$ (ITGAV and ITGB3) for fibronectin and vitronectin receptor, and $\alpha \mathrm{v} \beta 6$ (ITGAV and ITGB3) were determined by qRT-PCR. Each value represents the mean \pm SEM of three independent experiments. ${ }^{*} \mathrm{P}<0.05,{ }^{* *} \mathrm{P}<0.01$ vs. the TPA group.

various tumor types. The treatment with TPA for $1 \mathrm{~h}$ increased the expression of ITGAV and ITGB3 in a time-dependent manner. Although the TPA-induced gene expressions did not significantly changed $12 \mathrm{~h}$ after irradiation, proton beam irradiation remarkably decreased the gene expression of all subunits after $24 \mathrm{~h}$ (Fig. 2). Therefore, these findings suggest that proton beam can inhibit migration and invasion through the inhibition of the gene expression of members of the integrin family in TPA-induced HT-29 human colorectal adenocarcinoma cells.

Proton beam irradiation inhibits the phosphorylation of key molecules involved in integrin signaling. To investigate whether proton beam irradiation regulates integrin signaling pathways, we assessed the effects of proton beam irradiation on the phosphorylation status of key molecules involved in integrin signaling pathways 1 and 3 days after irradiation. Three days after irradiation, the phosphorylation of Src, a non-receptor tyrosine kinase, as well as p130Cas, an adaptor protein were consistently increased. The phosphorylation of FAK by TPA was increased 1 day after irradiation, but was decreased at 3 days after irradiation. Proton beam irradiation at 16 Gy completely suppressed the phosphorylation of the proteins (Fig. 3). Moreover, the phosphorylation of Src and p130Cas, but not FAK, was increased in the control group. These results suggest that proton beam irradiation may regulate cell adhesion and migration through the inhibition of the activities of downstream integrin signaling molecules.

Proton beam irradiation regulates the phosphorylation of molecules downstream of integrin signaling. To further study the regulatory mechanism underlying proton beam irradiation, we next investigated the phosphorylation of AMPK, PKC, and MAPK, which are molecules downstream of integrin signaling and well known for their roles in the regulation of various integrin-dependent cellular functions. As shown in Fig. 4, the treatment with TPA for $1 \mathrm{~h}$ consistently decreased the phosphorylation of AMPK as compared with that in the control group. However, proton beam irradiation at 8 and $16 \mathrm{~Gy}$ resulted in notable increases in the phosphorylation of AMPK. On the contrary, an inhibitory effect on the phosphorylation of PKC and MAPK was observed 3 days after irradiation 


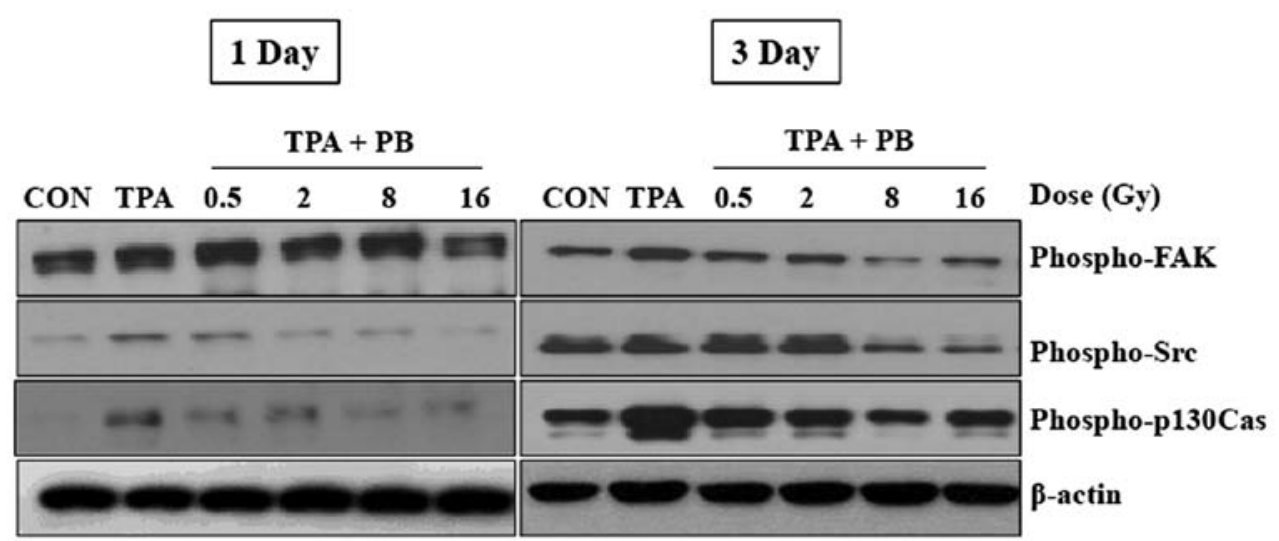

Figure 3. Effects of proton beam irradiation on the phosphorylation of FAK, c-Src, and p130Cas, which are related to integrin-mediated signaling pathways, in TPA-induced HT-29 human colorectal adenocarcinoma cells. Before irradiation, cells were treated with $150 \mathrm{nM}$ TPA for $1 \mathrm{~h}$. After irradiation with proton beam at different doses, cells were then incubated for $24 \mathrm{~h}$. Cells were reseeded and incubated for 1 and 3 days. Western blot analysis was performed with anti-phospho FAK, anti-phospho c-Src, and anti-phospho FAK antibodies. $\beta$-actin served as the loading control.

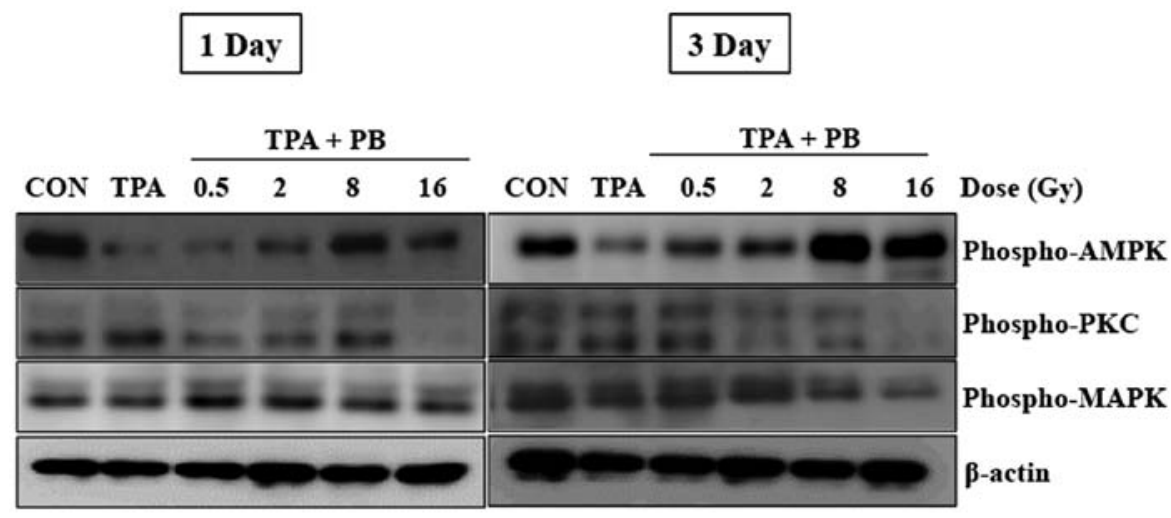

Figure 4. Effects of proton beam irradiation on the phosphorylation of AMPK, PKC, and MAPK, which are related to integrin-mediated cell adhesion and migration, in TPA-induced HT-29 human colorectal adenocarcinoma cells. Western blot analysis was performed with anti-phospho AMPK, anti-phospho PKC, and anti-phospho MAPK antibodies. $\beta$-actin served as the loading control.

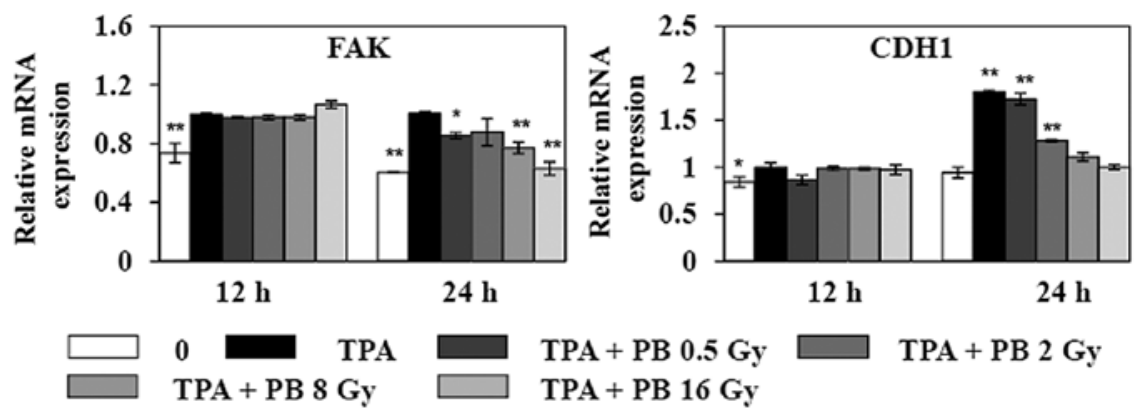

Figure 5. Effects of proton beam irradiation on the gene expression of FAK and E-cadherin, which are related to cell adhesion and migration, in TPA-induced HT-29 human colorectal adenocarcinoma cells. Before irradiation, cells were treated with $150 \mathrm{nM}$ TPA for $1 \mathrm{~h}$. After cells were irradiated with proton beam at different doses, expression of FAK and E-cadherin (CDH1) were determined by qRT-PCR. Each value represents the mean \pm SEM of three independent experiments. ${ }^{*} \mathrm{P}<0.05,{ }^{* *} \mathrm{P}<0.01$ vs. the TPA group.

at $16 \mathrm{~Gy}$. These results suggest that proton beam irradiation may regulate integrin-mediated functions by upregulating the activity of AMPK.

Proton beam irradiation inhibits the expressions of genes related to integrin-mediated cell adhesion and integrin trafficking. To study how proton beam irradiation affects integrin-mediated functions, we investigated the expression of genes that regulate integrin-mediated adhesion to ECM, as well as integrin trafficking. As shown in Fig. 5, the treatment with TPA increased the expressions of CDH1 and FAK $12 \mathrm{~h}$ after irradiation. Proton beam irradiation dose-dependently decreased the expressions of FAK and CDH1 $24 \mathrm{~h}$ after irradiation. To further study the effects of proton beam irradiation 

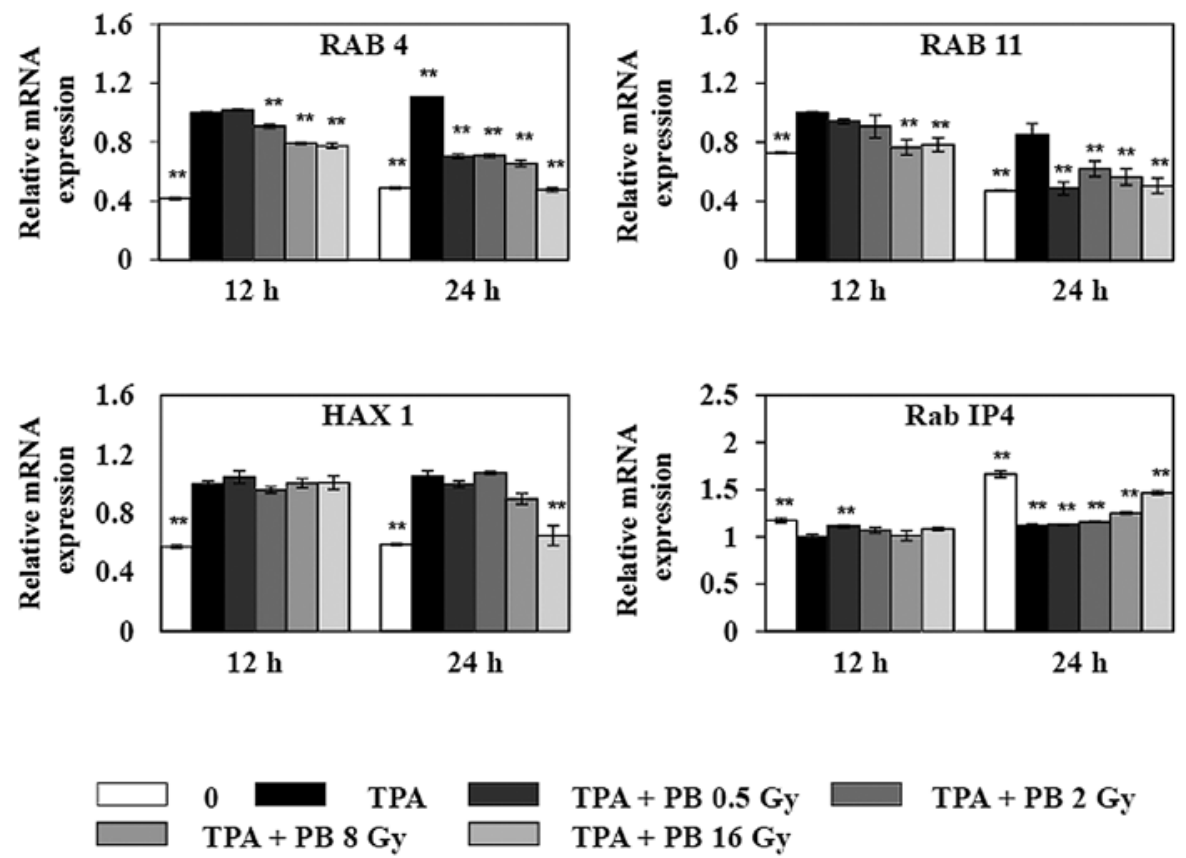

Figure 6. Effects of proton beam irradiation on the gene expressions of RAB4, RAB11, HAX1, and Rab IP4, which are related to integrin trafficking, in TPAinduced HT-29 human colorectal adenocarcinoma cells. Before irradiation, cells were treated with $150 \mathrm{nM}$ TPA for $1 \mathrm{~h}$. After cells were irradiated with proton beam at different doses, expressions of RAB4, RAB11, HAX1, and Rab IP4 were determined by qRT-PCR. Each value represents the mean \pm SEM of three independent experiments. ${ }^{*} \mathrm{P}<0.05,{ }^{* *} \mathrm{P}<0.01$ vs. the TPA group.

on integrin functions, we assessed the effect of proton beam irradiation on the expressions of genes related to integrin trafficking (Fig. 6). Proton beam irradiation dose-dependently decreased the gene expression of Rab family of small GTPases, such as RAB4, which mediates fast integrin recycling from the early endosome RAB11, which mediates slow integrin recycling through recycling endosome; and $\mathrm{HAX} 1$, which mediates clathrin-dependent endocytosis of integrin, $24 \mathrm{~h}$ after irradiation. In contrast, TPA decreased the gene expression of Rab IP4, which blocks integrin recycling, leading to inhibition of cell adhesion and cell spreading. However, the expression of Rab IP4 was increased by proton beam irradiation at $8 \mathrm{~Gy}$. Therefore, these results suggest that proton beam irradiation can modulate cell adhesion and migration, as well as integrin trafficking necessary for tumor progression.

\section{Discussion}

In the present study, we demonstrated that proton beam irradiation suppresses protein expression of integrin $\beta 1$, gene expressions of members of the integrin family, integrin-mediated signaling pathways involving FAK, Src, and p130Cas, the phosphorylation of kinases such as PKC and MAPK, and gene expression of regulators involved in integrin trafficking such as RAB4, RAB11, and HAX1, as well as increasing the phosphorylation of AMPK and Rab IP4, which are well known as negative regulators on tumor progression and metastasis.

Proton beam therapy (PBT) has recently attracted attention for its use as an alternative to gamma or X-ray irradiation therapy and been producing promising clinical results worldwide (23). PBT has been used for decades, mainly to treat hepatocellular carcinoma (24), non-small cell lung cancer (25), prostate cancer (26), and head and neck tumors (27). In addi- tion, many in vitro studies have shown that PBT suppresses metastatic capability, including adhesion and migration, in diverse human cancer cell lines (28-30). However, a more detailed investigation on the inhibitory effect of proton beam on metastatic potential of cancer cells is still needed.

Integrin $\beta 1$ is the most widely expressed integrin in cells, and it has been implicated in the clinical course and prognosis of several types of cancer (31). Integrin $\beta 1$ plays a significant role in tumor metastasis. It binds to ECM and initiates adhesion by recruiting cytoplasmic proteins, such as Src, FAK, and p130Cas (32). The multiple parallel signaling pathways downstream of integrin engagement that promote tumor growth include FAK, Src, PKC, MAP kinase, Akt, and Ras pathways. These pathways are upregulated as the level of integrin $\beta 1$ increases (33). Indeed, several studies have demonstrated the correlation of the expression of integrin $\beta 1$ with malignant features, including metastasis $(34,35)$. Accordingly, integrin $\beta 1$ signaling in tumor cells has been shown to promote resistance to multiple treatment modalities, including cytotoxic drugs, radiotherapy (36), and targeted therapies such as trastuzumab (37) and lapatinib (38). Studies using conditional genetic models point to critical roles of integrin $\beta 1$ in initiation, growth, or progression of a variety of cancers (4). In a prostate adenocarcinoma model, deletion of integrin $\beta 1$ led to more dramatic expansion of the tumor cell population, enhanced the rate of prostate tumor progression, and decreased overall animal survival (39).

The metastatic potential of tumors depends on integrin complexes, which function as intracellular signaling mediators. Integrins promote migration of cells on the surrounding ECM, and the signals initiated by integrin binding to ECM proteins are necessary for the maintenance of cell survival. Focal adhesion sites contain integrins and complexes of 
signaling elements, such as Src, FAK, p130Cas, MAP kinases, small GTPases, and phosphoinositide 3-kinase (40). Although it is not clear which regulatory mechanism is employed by proton beam irradiation to modulate the expression of integrin $\beta 1$, we demonstrated that proton beam irradiation suppressed the protein expression of integrin $\beta 1$, leading to an inhibition of the phosphorylated forms of FAK, Src, and p130Cas, which are molecules downstream of the integrin $\beta 1$ signaling pathway. The decrease in the expression of integrin $\beta 1$ protein was accompanied by changes in mRNA levels of integrin $\beta 1$ as well as other members of the integrin family, suggesting a post-translational mechanism. Therefore, our findings demonstrate that downregulation of the protein level of integrin $\beta 1$ and activities of downstream signaling molecules is a novel mechanism underlying the suppressive effect of proton beam irradiation on the migration of cancer cells.

On the contrary, recent studies have shown not only integrin signaling, but also integrin trafficking contribute to cancer growth and progression $(4,6)$. Abundant evidence suggests that integrin trafficking regulates cell adhesion to ECM, establishes and maintains cell polarity, redefines signaling pathways, and controls migration (41). Therefore, transcriptional changes, mutational alterations, and deregulated cellular signaling changing endocytosis and recycling of integrins confer invasive and metastatic properties to tumor cells. Although at least $24 \alpha \beta$ integrin heterodimers are known, $\alpha 5 \beta 1, \alpha 6 \beta 4, \alpha v \beta 3$, and $\alpha v \beta 6$ integrins have been extensively studied in cancer and the expression is correlated with cancer progression in various tumor types (7). Upregulation of these integrins renders cancer cells more motile, invasive, and resistant to anticancer drugs (42). Unlike these integrins, expression levels of certain integrins, such as $\alpha 2 \beta 1$ (43) and $\alpha 1 \beta 1$ (44), decrease in tumor cells, which potentially increase tumor cell dissemination. In addition to changes in expression, changes in the functions of these integrins also play a critical role in cancer progression. Therefore, our findings suggest proton beam irradiation as a general and strong inhibitor on the expression of members of the integrin family. AMPK is a metabolic sensor that maintains cellular energy homeostasis. AMPK regulates lipid, cholesterol, and glucose metabolism in specialized metabolic tissues, such as liver, muscle, and adipose tissues. This function has made AMPK a key therapeutic target in patients with obesity and diabetes (45). The connection of AMPK with several tumor suppressors suggests that therapeutic manipulation of this pathway using established diabetes drugs warrants further investigation in patients with cancer (46). Although previous studies showed that LKB1-deficient (47) or AMPKdeficient cells (48) are resistant to oncogenic transformation and tumorigenesis, the role of AMPK in tumorigenesis and tumor metabolism is unknown. Recent studies have indicated that AMPK controls metastasis of cancer cells $(49,50)$. Interestingly, our findings indirectly show that an increase in AMPK phosphorylation by proton beam irradiation may suppress metastatic potentials of TPA-induced HT-29 human colorectal adenocarcinoma cells. However, a more detailed investigation on the regulatory mechanism underlying proton beam irradiation-induced AMPK phosphorylation is still needed.

In conclusion, our findings suggest that proton beam irradiation can inhibit metastatic potential including cell adhesion and migration by modulating gene expression of integrins, genes involved in integrin trafficking, and activities of molecules involved in integrin sigmaling necessary for tumor progression.

\section{Acknowledgements}

This study was supported by the National Research Foundation of Korea (NRF) grant funded by the Korea government (MSIP) (NRF-2014M2B2A4030340).

\section{References}

1. Chaudhary AK, Pandya S, Ghosh K and Nadkarni A: Matrix metalloproteinase and its drug targets therapy in solid and hematological malignancies: An overview. Mutat Res 753: 7-23, 2013.

2. Ganguly KK, Pal S, Moulik S and Chatterjee A: Integrins and metastasis. Cell Adhes Migr 7: 251-261, 2013.

3. Cook DR, Rossman KL and Der CJ: Rho guanine nucleotide exchange factors: Regulators of Rho GTPase activity in development and disease. Oncogene 33: 4021-4035, 2014.

4. Xiong J, Balcioglu HE and Danen EH: Integrin signaling in control of tumor growth and progression. Int J Biochem Cell Biol 45: 1012-1015, 2013.

5. Kapp TG, Rechenmacher F, Sobahi TR and Kessler H: Integrin modulators: a patent review. Expert opinion on therapeutic patents 23: 1273-1295, 2013.

6. Shin S, Wolgamott L and Yoon SO: Integrin trafficking and tumor progression. Int J Cell Biol 2012: 516789, 2012.

7. Desgrosellier JS and Cheresh DA: Integrins in cancer: Biological implications and therapeutic opportunities. Nat Rev Cancer 10: 9-22, 2010.

8. Mitra SK and Schlaepfer DD: Integrin-regulated FAK-Src signaling in normal and cancer cells. Curr Opin Cell Biol 18: 516-523, 2006

9. Hahn SS, Tang Q, Zheng F, Zhao S, Wu J and Chen J: Repression of integrin-linked kinase by antidiabetes drugs through crosstalk of PPAR $\gamma$ - and AMPK $\alpha$-dependent signaling: Role of AP- $2 \alpha$ and Sp1. Cell Signal 26: 639-647, 2014.

10. Caino MC, Chae YC, Vaira V, Ferrero S, Nosotti M, Martin NM, Weeraratna A, O'Connell M, Jernigan D, Fatatis A, et al: Metabolic stress regulates cytoskeletal dynamics and metastasis of cancer cells. J Clin Invest 123: 2907-2920, 2013.

11. Wang J, Wu J, Hong J, Chen R, Xu K, Niu W, Peng C, Liu E, Wang J, Liu S, et al: PKC promotes the migration of colon cancer cells by regulating the internalization and recycling of integrin av 36. Cancer Lett 311: 38-47, 2011.

12. Chen J, Elfiky A, Han M, Chen C and Saif MW: The role of Src in colon cancer and its therapeutic implications. Clin Colorectal Cancer 13: 5-13, 2014.

13. Caswell PT and Norman JC: Integrin trafficking and the control of cell migration. Traffic 7: 14-21, 2006.

14. Hutagalung AH and Novick PJ: Role of Rab GTPases in membrane traffic and cell physiology. Physiol Rev 91: 119-149, 2011.

15. Subramani D and Alahari SK: Integrin-mediated function of Rab GTPases in cancer progression. Mol Cancer 9: 312, 2010.

16. Vukmirica J, Monzo P, Le Marchand-Brustel Y and Cormont M: The Rab4A effector protein Rabip4 is involved in migration of NIH 3T3 fibroblasts. J Biol Chem 281: 36360-36368, 2006.

17. Ramsay AG, Keppler MD, Jazayeri M, Thomas GJ, Parsons M, Violette S, Weinreb P, Hart IR and Marshall JF: HS1-associated protein $\mathrm{X}-1$ regulates carcinoma cell migration and invasion via clathrin-mediated endocytosis of integrin alphavbeta6. Cancer Res 67: 5275-5284, 2007.

18. Mitin T and Zietman AL: Promise and pitfalls of heavy-particle therapy. J Clin Oncol 32: 2855-2863, 2014.

19. Nam KS, Kim MK and Shon YH: Cancer chemopreventive enzymes of human colorectal adenocarcinoma cells irradiated with proton beams. J Korean Phys Soc 52: 945-948, 2008.

20. Nam KS and Shon YH: Suppression of metastatic potential in human colorectal adenocarcinoma cells irradiated with proton beams. J Korean Phys Soc 59: 709-712, 2011.

21. Lee KB, Kim KR, Huh TL and Lee YM: Proton induces apoptosis of hypoxic tumor cells by the p53-dependent and p38/JNK MAPK signaling pathways. Int J Oncol 33: 1247-1256, 2008 . 
22. Sikorsky JA, Primerano DA, Fenger TW and Denvir J: Effect of DNA damage on PCR amplification efficiency with the relative threshold cycle method. Biochem Biophys Res Commun 323: 823-830, 2004

23. Combs SE, Djosanjh M, Pötter R, Orrechia R, Haberer T, Durante M, Fossati P, Parodi K, Balosso J, Amaldi U, et al: Towards clinical evidence in particle therapy: ENLIGHT, PARTNER, ULICE and beyond. J Radiat Res (Tokyo) 54 (Suppl 1): i6-i12, 2013.

24. Lee SU, Park JW, Kim TH, Kim YJ, Woo SM, Koh YH, Lee WJ, Park SJ, Kim DY and Kim CM: Effectiveness and safety of proton beam therapy for advanced hepatocellular carcinoma with portal vein tumor thrombosis. Strahlenther Onkol 190: 806-814, 2014.

25. Chang JY, Komaki R, Lu C, Wen HY, Allen PK, Tsao A, Gillin M, Mohan R and Cox JD: Phase 2 study of high-dose proton therapy with concurrent chemotherapy for unresectable stage III nonsmall cell lung cancer. Cancer 117: 4707-4713, 2011.

26. Hoppe B, Henderson R, Mendenhall WM, Nichols RC, Li Z and Mendenhall NP: Proton therapy for prostate cancer. Oncology 25: 644-650, 652, 2011.

27. Frank SJ and Selek U: Proton beam radiation therapy for head and neck malignancies. Curr Oncol Rep 12: 202-207, 2010.

28. Gridley DS, Pecaut MJ, Mao XW, Wroe AJ and Luo-Owen X Biological effects of passive versus active scanning proton beams on human lung epithelial cells. Technol Cancer Res Treat 14: 81-98, 2015.

29. Wéra AC, Heuskin AC, Riquier H, Michiels $\mathrm{C}$ and Lucas $\mathrm{S}$ : Low-LET proton irradiation of A549 non-small cell lung adenocarcinoma cells: Dose response and RBE determination. Radiat Res 179: 273-281, 2013

30. Zaboronok A, Isobe T, Yamamoto T, Sato E, Takada K, Sakae T, Tsurushima $\mathrm{H}$ and Matsumura A: Proton beam irradiation stimulates migration and invasion of human U87 malignant glioma cells. J Radiat Res (Tokyo) 55: 283-287, 2014.

31. Brakebusch $\mathrm{C}$ and Fässler R: beta 1 integrin function in vivo: Adhesion, migration and more. Cancer Metastasis Rev 24: 403-411, 2005.

32. Canel M, Serrels A, Frame MC and Brunton VG: E-cadherinintegrin crosstalk in cancer invasion and metastasis. J Cell Sci 126: 393-401, 2013

33. Jahangiri A, Aghi MK and Carbonell WS: $\beta 1$ integrin: Critical path to antiangiogenic therapy resistance and beyond. Cancer Res 74: 3-7, 2014.

34. Parvani JG, Galliher-Beckley AJ, Schiemann BJ and Schiemann WP: Targeted inactivation of $\beta 1$ integrin induces $\beta 3$ integrin switching, which drives breast cancer metastasis by TGF- $\beta$. Mol Biol Cell 24: 3449-3459, 2013.

35. Howe GA and Addison CL: $\beta 1$ integrin: An emerging player in the modulation of tumorigenesis and response to therapy. Cell Adhes Migr 6: 71-77, 2012.

36. Nam JM, Chung Y, Hsu HC and Park CC: betal integrin targeting to enhance radiation therapy. Int J Radiat Biol 85 : 923-928, 2009.
37. Lesniak D, Sabri S, Xu Y, Graham K, Bhatnagar P, Suresh M and Abdulkarim B: Spontaneous epithelial-mesenchymal transition and resistance to HER-2-targeted therapies in HER-2-positive luminal breast cancer. PLoS One 8: e71987, 2013.

38. Huang C, Park CC, Hilsenbeck SG, Ward R, Rimawi MF, Wang YC, Shou J, Bissell MJ, Osborne CK and Schiff R: $\beta 1$ integrin mediates an alternative survival pathway in breast cancer cells resistant to lapatinib. Breast Cancer Res 13: R84, 2011.

39. Moran-Jones K, Ledger A and Naylor MJ: $\beta 1$ integrin deletion enhances progression of prostate cancer in the TRAMP mouse model. Sci Rep 2: 526, 2012.

40. Harburger DS and Calderwood DA: Integrin signalling at a glance. J Cell Sci 122: 159-163, 2009.

41. Caswell PT, Vadrevu S and Norman JC: Integrins: Masters and slaves of endocytic transport. Nat Rev Mol Cell Biol 10: 843-853, 2009.

42. Makrilia N, Kollias A, Manolopoulos L and Syrigos K: Cell adhesion molecules: Role and clinical significance in cancer. Cancer Invest 27: 1023-1037, 2009.

43. Ramirez NE, Zhang Z, Madamanchi A, Boyd KL, O'Rear LD, Nashabi A, Li Z, Dupont WD, Zijlstra A and Zutter MM: The $\alpha(2) \beta(1)$ integrin is a metastasis suppressor in mouse models and human cancer. J Clin Invest 121: 226-237, 2011.

44. Mattila E, Pellinen T, Nevo J, Vuoriluoto K, Arjonen A and Ivaska J: Negative regulation of EGFR signalling through integrin-alphalbeta1-mediated activation of protein tyrosine phosphatase TCPTP. Nat Cell Biol 7: 78-85, 2005.

45. Grahame Hardie D: AMP-activated protein kinase: A key regulator of energy balance with many roles in human disease. J Intern Med 276: 543-559, 2014.

46. Shackelford DB and Shaw RJ: The LKB1-AMPK pathway: Metabolism and growth control in tumour suppression. Nat Rev Cancer 9: 563-575, 2009.

47. Bardeesy N, Sinha M, Hezel AF, Signoretti S, Hathaway NA, Sharpless NE, Loda M, Carrasco DR and DePinho RA: Loss of the Lkb1 tumour suppressor provokes intestinal polyposis but resistance to transformation. Nature 419: 162-167, 2002.

48. Kato K, Ogura T, Kishimoto A, Minegishi Y, Nakajima N, Miyazaki M and Esumi H: Critical roles of AMP-activated protein kinase in constitutive tolerance of cancer cells to nutrient deprivation and tumor formation. Oncogene 21: 6082-6090, 2002.

49. Chan KT, Asokan SB, King SJ, Bo T, Dubose ES, Liu W, Berginski ME, Simon JM, Davis IJ, Gomez SM, et al: LKB1 loss in melanoma disrupts directional migration toward extracellular matrix cues. J Cell Biol 207: 299-315, 2014.

50. Goodwin JM, Svensson RU, Lou HJ, Winslow MM, Turk BE and Shaw RJ: An AMPK-independent signaling pathway downstream of the LKB1 tumor suppressor controls Snaill and metastatic potential. Mol Cell 55: 436-450, 2014. 